\section{PUERTO MADERO 'EN MOVIMIENTO': MOVILIDAD DE POLÍTICAS Y MODELOS URBANOS EN AMÉRICA LATINA (1999-2012) ${ }^{1}$}

Guillermo Paz Jajamovich²

\section{Resumen}

En las últimas décadas, se intensificaron los viajes, la transferencia y el flujo de los modelos urbanos, políticas urbanas, ideas y técnicas y se acrecentó el interés académico en tales procesos. Paralelamente, en América Latina, se han multiplicado tanto los grandes proyectos urbanos como su análisis. Este artículo busca conectar ambas cuestiones a partir del abordaje de la circulación de la Corporación Antiguo Puerto Madero Sociedad Anónima (CAPMSA) en distintas ciudades latinoamericanas interrogando cómo se movilizan los modelos y políticas urbanas, a través de qué circuitos de políticas
PUERTO MADERO 'IN MOTION': POLICY MOBILITY AND URBAN MODELS IN LATIN AMERICA (1999-2012)

Guillermo Paz Jajamovich²

\title{
Abstract
}

The last few decades have seen an increase in the travel and transference of urban models and policies, ideas and techniques as well as an increased academic interest in these processes. At the same time, Latin America has experienced a proliferation of large urban projects and relevant analysis. This paper seeks to establish a link between these two issues by addressing the work of the Corporación Antiguo Puerto Madero Sociedad Anónima (CAPMSA) in different Latin American cities and raising questions about the mobilization of urban models and policies, the mechanisms for the mobilization of these 
y quiénes son los expertos y actores sociales que las hacen circular.

\section{PALABRAS CLAVE: CORPORACIÓN ANTIGUO PUERTO MADERO; CIRCULACIÓN DE POLÍTICAS URBANAS; GRANDES PROYECTOS URBANOS.}

initiatives and the experts and social actors in charge of the circulation of these mechanisms.

\section{KEYWORDS: CORPORACIÓN ANTIGUO PUERTO MADERO; URBAN POLICY MOBILITY; LARGE URBAN PROJECTS.}

Recibido: 19-12-2014

Aceptado: 18-01-2016

1 Este trabajo es producto de un Proyecto de Investigación Plurianual (PIP) - Grupo de Investigación - 2014-2016 / nro. $11220130100717 C 0$, titulado “La circulación regional de políticas y modelos urbanos: articulaciones público-privadas en la expansión latinoamericana de la Corporación Antiguo Puerto Madero S.A (1999-2013)". Se trata de un proyecto financiado por el Consejo Nacional de Investigaciones Científicas y Técnicas con sede en el Instituto de Estudios de América Latina y el Caribe, Facultad de Ciencias Sociales, Universidad de Buenos Aires.

2 Argentina. Doctor en Ciencias Sociales. Investigador asistente del Consejo Nacional de Investigaciones Científicas y Técnicas con sede en el Instituto de Estudios de América Latina y el Caribe, Facultad de Ciencias Sociales, Universidad de Buenos Aires. Profesor visitante de Maestría en Historia y Cultura de la Arquitectura y la Ciudad en la Universidad Torcuato Di Tella. Correo electrónico: guillermopazjajamovich@gmail.com.
Received: 19-12-2014

Accepted: 18-01-2016

1 This paper is part of a Multiannual Research Project (PIP) - Research Group - 2014-2016 No 11220130100717CO “Regional Dissemination of Urban Policies and Models: Public-Private Relationships within the Context of the Latin American Expansion of the Corporación Antiguo Puerto Madero S.A (1999-2013)". This is a project funded by the National Scientific and Technical Research Council at the Institute of Latin American and Caribbean Studies, Faculty of Social Sciences, University of Buenos Aires.

2 Argentina. PhD in Social Sciences. Assistant researcher, National Scientific and Technical Research Council at the Institute of Latin American and Caribbean Studies, Faculty of Social Sciences, University of Buenos Aires. Visiting professor at the University Torcuato Di Tella, Master's program in History and Culture of Architecture and the City. Email: guillermopazjajamovich@gmail.com. 


\section{Introducción}

Las últimas décadas han sido caracterizadas como un período de profundas transformaciones a nivel urbano, observando cambios morfológicos y económicos, modificaciones en las políticas urbanas y en los modos de intervenir arquitectónicamente, mutaciones en las maneras de conceptualizar la ciudad y en los roles que se le otorgan, entre otras cuestiones. Esto se condice con un proceso internacional de crisis de un ciclo "expansivo" o "desarrollista" a partir de múltiples procesos tales como la deslocalización industrial, el fin del crecimiento demográfico urbano por migraciones, la proliferación de espacios insulares, las críticas al urbanismo moderno y la emergencia de nuevas formas de intervenir en la ciudad ${ }^{3}$. Asimismo, este proceso de transformación urbana se vincula estrechamente con reestructuraciones económicas y con el rol del Estado que se producen a un nivel internacional. Ello implicó cambios en el rol planificador propio del Estado de bienestar, sus modos de intervenir y la articulación de lo público y lo privado. En ese marco, han proliferado nuevos modos de hacer ciudad, caracterizados por

3 Ciccolella, 1999; Menazzi, 2013. intervenciones más puntuales y fragmentarias, en articulación con el capital privado. Aquellas nuevas formas de intervenir y gestionar la ciudad se han ido difundiendo y multiplicando en gran cantidad de ciudades. Así, un relevante corpus de trabajos ha vinculado estos aspectos con procesos como los de globalización ${ }^{4}$. De modo complementario, un creciente grupo de investigaciones indica que la simultaneidad de formas análogas de intervenir en la ciudad no se deriva mecánicamente de los procesos de globalización sino que suponen el accionar de múltiples redes de actores -y una serie de procesos- más específicos ${ }^{5}$.

Una de las iniciativas que más se vinculó a estos nuevos modos de hacer ciudad en el caso de Buenos Aires fue la urbanización de Puerto Madero, cuya gestión estuvo a cargo de la Corporación Antiguo Puerto Madero S.A., CAPMSA ${ }^{6}$. Tanto la CAPMSA como diversos actores se han encargado de dar a conocer este proyecto por diversos medios y han abordado distintas aristas de tal operación ${ }^{7}$. Asimismo, desde el universo académico se han producido numerosos estudios acerca de esta intervención ${ }^{8}$. Sin embargo, poco se conoce

\footnotetext{
4 Sassen, 1999.

5 Jajamovich, 2013; Novick, 2009

6 De aquí en adelante nos referiremos a la Corporación Antiguo Puerto Madero como CAPMSA o Corporación.

7 Garay, 2007; Corporación Antiguo Puerto Madero, 1999 y 2011.

8 Gorelik, 2007; Cuenya y Corral, 2011; Jajamovich, 2012; Etulain, 2008.
} 
en relación al accionar de la Corporación por fuera del perímetro de las 170 hectáreas que comprende el área de Puerto Madero. En efecto, desde 1999, ésta funciona como un ente que promueve y asesora sobre operaciones urbanas en múltiples ciudades de la región.

Este trabajo busca analizar el accionar de la Corporación en el marco de otros campos problemáticos, como la temática de la circulación internacional y la movilidad de técnicos, modelos, políticas e ideas urbanas. Asimismo, a partir de tal abordaje, se busca dialogar con recientes investigaciones sobre grandes proyectos urbanos en América Latina.

Partiendo de la idea de que las políticas y modelos urbanos no circulan en cualquier dirección sino que suponen el accionar de diversos actores interesados en movilizar tales procesos, este artículo resalta la utilidad metodológica del análisis de trayectorias de expertos para entender cómo y hacia dónde circulan los modelos y las políticas urbanas así como para dar cuenta de la multiplicidad de caminos que toma tal movilidad y la variedad de escalas que atraviesa. Como se verá, esto supone un desplazamiento desde perspectivas que construyen explicaciones más estructurales hacia otras que sin dejar de lado tales aspectos, buscan complementarlas con el abordaje de otros procesos, favoreciendo una mirada más enfocada en los actores $^{9}$. Como consecuencia de este giro, este artículo subraya la necesidad de atender la relación entre la CAPMSA y sus integrantes, tanto cuando éstos trabajan en el marco de la Corporación como cuando lo hacen fuera de la misma, diversificando y multiplicando la movilidad de las políticas y modelos urbanos y dificultando una delimitación clara de la expansión de la Corporación. Asimismo, esto supone la consideración de múltiples yuxtaposiciones entre actores públicos y privados para dar cuenta de tales procesos ${ }^{10}$. De todos modos, tal como se desarrollará, el abordaje de las trayectorias de los integrantes de la Corporación no agota la movilidad y circulación de experiencias como la de Puerto Madero, en tanto ésta también reconoce formas más lábiles de expansión.

9 Múltiples investigadores resaltan la importancia de dar cuenta del rol de los actores -y sus relaciones- en la circulación de políticas urbanas. Jacobs y Lees, 2013; Healey, 2013; Nasr y Volait, 2003.

10 El abordaje se apoya en diversas fuentes como los balances anuales de la CAPMSA -desde el año 2001 hasta el año 2012 -, publicaciones de la misma y su página web. Además, hemos realizado entrevistas en profundidad a integrantes de la Corporación, así como han sido analizadas diversas fuentes secundarias vinculadas a la temática. 
El presente trabajo se organiza del siguiente modo: en primer lugar, aborda diversas perspectivas que analizan la circulación y movilidad de las políticas y modelos urbanos y retoma una serie de planteos a propósito del abordaje de grandes proyectos urbanos en América Latina. En segundo término, analiza el momento y el modo en que la CAPMSA empieza a funcionar como una corporación que no sólo se propone concluir la urbanización de Puerto Madero, sino que inicia su camino en la promoción de emprendimientos en ciudades argentinas y latinoamericanas. En tercer lugar, se indican algunas observaciones sobre los convenios e iniciativas en que ha participado la CAPMSA fuera de los límites de Puerto Madero y se interrogan las formas cambiantes que ha asumido su participación. Asimismo, se señalan otras modalidades de circulación más difusas de la Corporación que permiten acercarse a entender cómo y a través de qué formas, diversos políticos y expertos de otras ciudades se acercan a la misma. Por último, se concluye retomando aspectos previamente indicados.

\section{Perspectivas para abordar la movilidad y la circulación de políticas y modelo urbanos}

El abordaje de la movilidad, circulación y transferencia de modelos y políticas urbanas entre distintas ciudades excede las fronteras nacionales. Al analizar cómo la CAPMSA circula entre distintos espacios, se dialoga con el creciente interés académico en los viajes, la transferencia y el flujo de los modelos urbanos, políticas urbanas, ideas y técnicas, así como con una serie de abordajes respecto a los grandes proyectos urbanos en América Latina.

Respecto al primer campo temático, Harris y Moo$\mathrm{re}^{11}$ indican que aquel interés académico se ha acrecentado en la última década reflejando la propia aceleración e intensificación de la circulación trasnacional de ideas, planes y políticas urbanas. Asimismo, señalan que tal interés se ha materializado en el análisis de una variedad de temas y formas espaciales y que tales aspectos son analizados por diversas disciplinas y campos de estudios a través de múltiples conceptos, como: policy mobility $^{12}$, urban learning assemblage ${ }^{13}$, policies in motion $^{14}$, spaces of circulation knowledge ${ }^{15}$, entre otros.

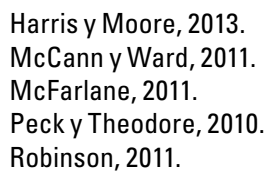


En ese plano, diversos investigadores señalan la imposibilidad de continuar analizando la producción de políticas en marcos estrictamente nacionales, ni en términos de traslación mecánica de lo global a lo local. Así, Cochrane y Ward ${ }^{16}$ proponen su abordaje en términos tanto relacionales como territoriales. Otros investigadores han insistido en la necesidad de una ampliación geográfica de los estudios del planeamiento urbano: Nasr y Volait ${ }^{17}$ han indicado la variedad de formas en que actores locales y extranjeros interactúan. Por su parte, Healey y Upton ${ }^{18}$ interrogan cómo las ideas se desplazan geográficamente, se incrustan y son modificadas por factores contextuales locales.

Si bien tales discusiones han sido mayormente producidas en el mundo anglosajón, cabe señalar la existencia de un creciente corpus de trabajos que analizan procesos de circulación de políticas y modelos urbanos y técnicos en América Latina. Más cercanos a la temática y periodo abarcados en este trabajo pueden mencionarse abordajes sobre: la circulación de la planificación estratégica urbana a partir del accionar de expertos catalanes y su implementación en Río de Janeiro ${ }^{19}$; la circulación del presupuesto participativo ${ }^{20}$ y las propuestas de

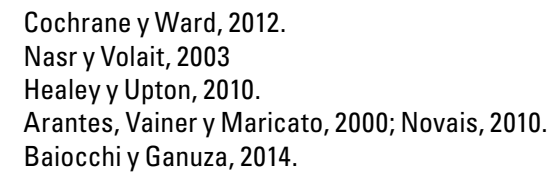

descentralización política ${ }^{21}$; la circulación de ciudades modelos' - como el caso de Curitiba-22, entre otros trabajos. En síntesis, como lo demuestran diversas investigaciones, los procesos de movilidad y circulación internacional de políticas y modelos urbanos no se producen únicamente desde el 'centro' hacia la 'periferia'. Así, el abordaje de la circulación y la movilidad de la CAMPSA permite ampliar un creciente grupo de trabajos que analiza la circulación y movilidad de las políticas urbanas entre ciudades del Sur.

Por otro lado, tal cual ya se indicó, este trabajo dialoga con abordajes recientes sobre los grandes proyectos urbanos en América Latina ${ }^{23}$. Es decir, operaciones de renovación urbana en gran escala que producen al menos tres modificaciones claves en la estructura de la centralidad de las actuales metrópolis: una modificación en la rentabilidad de los usos del suelo; una modificación funcional y físico espacial de áreas centrales estratégicas; y una modificación de los mecanismos de gestión pública ${ }^{24}$. En términos genéricos, en el abordaje de GPUs ha predominado el estudio de casos y el foco en sus efectos urbanos, sociales, culturales, políticos y/o económicos. En menores oportunidades se ha dado cuenta de la multiplicación de estas formas

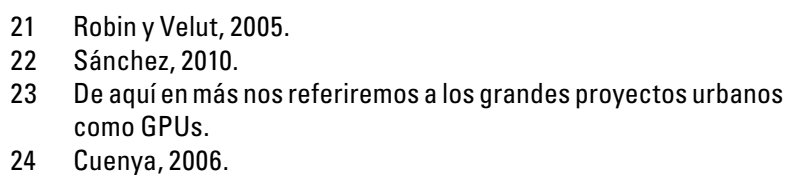


de intervención urbana en la región y cuando sí se tuvo en cuenta tal asunto, el eje estuvo centrado en indicar el accionar del capital transnacional sobre las ciudades. Intentando ampliar el panorama, este trabajo reconsidera aspectos trasnacionales de otro modo, dando cuenta de otros actores y procesos que intervienen en la multiplicación de intervenciones similares en diversas ciudades. En efecto, las ciudades apelan a una serie de recursos en los procesos de desarrollo y legitimación de estrategias de desarrollo urbano y políticas urbanas que incluyen la circulación internacional de expertos y de políticas urbanas ${ }^{25}$. Así, el abordaje de tales procesos permite la construcción de objetos de investigación trasnacionales, la puesta en diálogo de múltiples intervenciones urbanas en la región y el abordaje de alguna de sus conexiones concretas. Asimismo, permite construir más mediaciones entre el accionar del capital global y las intervenciones urbanas, subrayando el rol del accionar y la circulación de expertos y políticas urbanas. En efecto, si bien la materialización de estas intervenciones urbanas supone la participación de capitales privados, entender cómo y en qué direcciones circulan tales propuestas requiere la consideración de otros procesos y actores involucrados.

25 Mc Cann, 2011.
Esto último puede reiterarse respecto a abordajes sobre la operación Puerto Madero. Así, usualmente, ésta fue abordada en relación a procesos de globalización y reconversión urbana ${ }^{26}$. En otras ocasiones, centralmente a partir del foco en sus inicios, la operación fue analizada en relación a la circulación de técnicos y modelos urbanos ${ }^{27}$ así como a partir de las trayectorias de los técnicos y profesionales envueltos en la misma y los intercambios más amplios - políticos y económicos-entre locales y extranjeros ${ }^{28}$. Por otro lado, diversos trabajos han analizado proyectos que se vinculan a la expansión y circulación de la CAPMSA -aunque sin tematizar la problemática que se busca desplegar en este trabajo-, como el caso MendozaMadero $^{29}$, el Proyecto Retiro ${ }^{30}$, Posadas-Encarnación ${ }^{31}$, entre otros. En síntesis, la operación Puerto Madero ha sido analizada en profundidad desde diversos ejes. Sin embargo, el abordaje de la circulación de la CAPMSA fuera del área de Puerto Madero es un aspecto apenas señalado y pendiente de ser abordado a partir de perspectivas como la aquí presentada. 


\section{Cambios y continuidades en la CAPMSA}

La CAPMSA surge en el año 1989 a través de un convenio entre representantes del Ministerio de Obras y Servicios Públicos, del Ministerio del Interior, en representación del Poder Ejecutivo Nacional y la Intendencia Municipal. Su objetivo era: "...la confección de un plan maestro de desarrollo urbano, el estudio de la infraestructura a incorporar y la promoción de inversiones en el área, la actividad inmobiliaria y la construcción de obras nuevas y/o remodelaciones en la zona de las obras necesarias para convertirla en un polo de desarrollo urbano basado en una genuina inversión, con participación de capitales nacionales y extranjeros, como asimismo la venta y/o locación de las tierras pertenecientes al área en cuestión..."32.

Constituida en 1989 como sociedad comercial, bajo la forma de Sociedad Anónima, sus accionistas son el Estado Nacional, dueño inicial de la tierra, y el Gobierno de la Ciudad de Buenos Aires, encargado de otorgarle normativa a la misma. El modelo de gestión que desarrolla la operación Puerto Madero

Poder Ejecutivo Nacional, 1989, p. 1. suponía una lógica de actuación público-privada. Asimismo, tal modelo de gestión debía permitir el autofinanciamiento a partir de la venta de tierra urbana y una urbanización por etapas, captando en cada nueva venta la valorización obtenida del proceso previo de urbanización. Es decir, la CAPMSA no contaba con presupuesto asignado sino solamente con las tierras. Surgida en un contexto nacional de reformas económicas y políticas neoliberales, como las leyes de reforma del Estado y la privatización de empresas públicas, el desarrollo de Puerto Madero era parte, en el contexto local, de un programa de privatizaciones ${ }^{33}$.

Inicialmente, la existencia y el accionar de la CAPMSA se circunscribían a la urbanización de las 170 hectáreas del área de Puerto Madero. Sin embargo, hacia 1999, "con la intención de proyectar las capacidades de la Sociedad, obtenidas en la exitosa experiencia urbanística de Puerto Madero"34, se amplía el objeto social de la Sociedad, "permitiéndole actuar como gerenciadora, desarrolladora, fiduciaria o inmobiliaria en otros proyectos de desarrollo inmobiliario y urbanístico, tanto

33 Mignaqui y Elguezabal, 1997.

34 Corporación Antiguo Puerto Madero, 2008, p. 23. 
dentro como fuera del país"35. De esta manera, la ampliación del objeto social de la Corporación es un modo de asegurar su continuidad institucional más allá del límite temporal y físico de la urbanización del área de Puerto Madero. Esa ampliación de facultades pone en escena a un nuevo actor que interviene en múltiples escalas (locales, nacionales y regionales) en los procesos de construcción de las ciudades ${ }^{36}$.

Así, en posteriores publicaciones propias de la CAPMSA, donde se remarcan sus logros tanto en el área de Puerto Madero como en su expansión internacional, ésta se presentará a sí misma como la protagonista de una fórmula exitosa factible de contribuir a partir de sus aprendizajes con otras ciudades que busquen generar proyectos afines: "Consolidados a nivel internacional como los realizadores de uno de los proyectos urbanos más exitosos de América Latina, la institución se propone contribuir a la formulación y montaje de nuevos proyectos urbanos. La cantidad de lecciones aprendidas durante estos veinte años le permite poner a disposición de emprendedores y gobiernos locales, equipos de profesionales con capacidad, conocimientos y experiencia para desarrollar este tipo de

35 Ibíd. Si bien la CAPMSA inicia este proceso hacia 1999, esto no implica que previamente no haya mantenido conversaciones más informales respecto a su experiencia con representantes técnicos y políticos de otras ciudades, como ser los casos de Montevideo (Uruguay) y Rosario (Argentina), entre otros. proyectos. Con esta actividad, la Corporación Antiguo Puerto Madero S.A. busca ayudar a emprendedores y gobiernos locales a concretar ideas e iniciativas que parecen de difícil implementación"137.

\section{La CAPMSA y su circulación}

\section{CONVENIOS E INICIATIVAS CON DIVERSAS CIUDADES}

La CAPMSA ha sido parte de diversas iniciativas en América Latina. Sin embargo, cabe indicar que gran parte de los convenios en los que participa no se materializaron, sea por no superar la etapa de convenios de intención, por su carácter de estudios de prefactibilidad, por la extensa temporalidad que su materialización demanda, por la resistencia de actores locales a los mismos o por conflictos entre reparticiones estatales. De todos modos, sí dan cuenta de la expansión, circulación y movilidad de la Corporación por fuera del área de Puerto Madero.

Los convenios e iniciativas en los que interviene la Corporación son heterogéneos. Así, existen

37 Corporación Antiguo Puerto Madero, 2011, p. 9. 
diferencias respecto al tamaño de las operaciones, al tipo de propuesta, la forma de participación de la CAPMSA, su ubicación geográfica, los comitentes y los socios, entre otros aspectos. La participación de la CAPMSA incluye propuestas de desarrollo conceptual, gerenciamiento de proyectos, asistencia técnica, elaboración de diagnósticos, estudios de prefactibilidad técnica, etc. Asimismo, la Corporación es requerida sucesivamente por su expertise en una variedad de cuestiones, en tanto 'modelo' de ciudad, de (auto)financiamiento, de articulación entre actores públicos y privados, de articulación interjurisdiccional, de recuperación de áreas centrales, de revitalización de áreas portuarias, etc. Su participación y grado de involucramiento varía en los distintos casos: en algunas ocasiones se trata de convenios y cartas de intención; en otras, de concursos y licitaciones con diverso grado de concreción. En algunas oportunidades la CAPMSA obtiene un rol protagónico, mientras en otras ocasiones es sólo un actor más en el marco de un entramado más amplio.

Si consideramos la geografía de la expansión de la CAPMSA, cabe indicar que de la totalidad de convenios e iniciativas en los que participa, predominan los contactos con ciudades argentinas, como la ciudad de Buenos Aires (tabla 1), y otras provincias del país, tales como: Mendoza, Corrientes, Misiones y Chaco (tabla 2). En menor medida, se despliegan convenios e iniciativas con ciudades latinoamericanas como Encarnación, Asunción, Santiago de Chile, Barranquilla, Río de Janeiro y Santo Domingo (tabla 3). Asimismo, mayoritariamente a partir de modalidades como las de concurso, pueden señalarse una serie de ciudades y países fuera de la región como Memphis, Osaka e Irlanda (tabla 4). 


\section{TABLA 1. LA EXPANSIÓN DE LA CORPORACIÓN ANTIGUO PUERTO MADERO: INICIATIVAS EN ÁREAS ANEXAS A PUERTO MADERO ${ }^{38}$.}

\begin{tabular}{|c|c|c|c|c|c|c|}
\hline Iniciativas & Año & Ubicación & Comitente / Socios & Tipo de acuerdo & Tamaño & Tareas \\
\hline Isla Demarcchi & 2001 & $\begin{array}{l}\text { Ciudad Autóno- } \\
\text { ma de Buenos } \\
\text { Aires (CABA) }\end{array}$ & $\begin{array}{l}\text { Presidencia de la Nación } \\
\text { - Gobierno de la Ciudad } \\
\text { Autónoma de Buenos Aires } \\
\text { (GCBA) }\end{array}$ & $\begin{array}{l}\text { Carta de } \\
\text { intención }\end{array}$ & 60 ha. & $\begin{array}{l}\text { Identificar inmuebles } \\
\text { y recopilar antece- } \\
\text { dentes para promover } \\
\text { integración al éjido de } \\
\text { la CABA }\end{array}$ \\
\hline $\begin{array}{l}\text { Conexión Puer- } \\
\text { to Madero-La } \\
\text { Boca }\end{array}$ & 2001 & CABA & Corporación Sur & Convenio & & $\begin{array}{l}\text { Lineamientos para } \\
\text { recuperación, puesta } \\
\text { en valor y continua- } \\
\text { ción del eje Sur }\end{array}$ \\
\hline Proyecto Retiro & 2001 & CABA & $\begin{array}{l}\text { Gobierno Nacional (GN)- } \\
\text { GCBA. Consultores: } \\
\text { Arqs. Varas - Lestard } \\
\text { - Baudizzone. }\end{array}$ & $\begin{array}{l}\text { Requerimiento } \\
\text { del GN y del } \\
\text { GCBA }\end{array}$ & 17,3 ha. & $\begin{array}{l}\text { Gerenciamiento del } \\
\text { Proyecto Plataforma } \\
\text { intermodal de trans- } \\
\text { porte de pasajeros } \\
\text { 'Retiro } 2010^{\prime}\end{array}$ \\
\hline $\begin{array}{l}\text { Localización } \\
\text { del Puerto de } \\
\text { Cruceros }\end{array}$ & 2006 & CABA & $\begin{array}{l}\text { Secretaría de Planeamiento } \\
\text { Urbano y GCBA }\end{array}$ & & & $\begin{array}{l}\text { Estudio preliminar } \\
\text { de prefactibilidad } \\
\text { técnica para emplaza- } \\
\text { miento de programa } \\
\text { turístico-portuario }\end{array}$ \\
\hline $\begin{array}{l}\text { Fundación La } \\
\text { Boca }\end{array}$ & 2007 & CABA & $\begin{array}{l}\text { Fundación La Boca-GN } \\
\text { - GCBA }\end{array}$ & $\begin{array}{l}\text { Integra Consejo } \\
\text { Fundación La } \\
\text { Boca }\end{array}$ & & $\begin{array}{l}\text { Programas de recupe- } \\
\text { ración de La Boca y la } \\
\text { Zona Sur, desde lo so- } \\
\text { cial y lo arquitectónico }\end{array}$ \\
\hline
\end{tabular}

Fuente: elaboración propia a partir de balances de la Corporación Antiguo Puerto Madero (2001 al 2012), publicaciones de la misma (Corporación Antiguo Puerto Madero, 2011) y artículos de diarios nacionales y locales.

38 Los espacios que no han sido llenados en las tablas se debe a la falta de fuentes adecuadas y/o a la imprecisión de las fuentes existentes respecto a las áreas aludidas. 


\section{TABLA 2. LA EXPANSIÓN DE LA CORPORACIÓN ANTIGUO PUERTO MADERO: INICIATIVAS EN PROVINCIAS DE ARGENTINA.}

\begin{tabular}{|c|c|c|c|c|c|c|}
\hline Iniciativas & Año & Ubicación & Comitente / Socios & Tipo de acuerdo & Tamaño & Tareas \\
\hline $\begin{array}{l}\text { Proyecto Potrerillos } \\
\text { - Madero }\end{array}$ & 2003 & Mendoza & Provincia de Mendoza & $\begin{array}{l}\text { Acuerdo } \\
\text { asociativo }\end{array}$ & 2000 ha. & $\begin{array}{l}\text { Establecer el marco jurídico para el } \\
\text { desarrollo de proyectos de inversión } \\
\text { privada y pública }\end{array}$ \\
\hline Proyecto Maipú & 2003 & Mendoza & Municipalidad de Maipú & $\begin{array}{l}\text { Solicitud de firma } \\
\text { de convenio }\end{array}$ & 43 ha. & $\begin{array}{l}\text { Desarrollar proyecto de Parque } \\
\text { Industrial, en predio de propiedad } \\
\text { municipal }\end{array}$ \\
\hline Proyecto San Rafael & 2003 & Mendoza & Municipalidad de San Rafael & $\begin{array}{l}\text { Solicitud de firma } \\
\text { de convenio }\end{array}$ & & $\begin{array}{l}\text { Desarrollo urbanístico de Estación } \\
\text { Terminal de Ómnibus de San Rafael } \\
\text { y dependencias accesorias }\end{array}$ \\
\hline $\begin{array}{l}\text { Proyecto Mendoza } \\
\text { - Madero }\end{array}$ & 2003 & Mendoza & $\begin{array}{l}\text { Convenio CAPMSA - Muni- } \\
\text { cipalidad de Mendoza. GN } \\
\text { - ONABE. }\end{array}$ & $\begin{array}{l}\text { Convenio, } \\
\text { creación de } \\
\text { comisión mixta y } \\
\text { fideicomiso }\end{array}$ & 36 ha. & $\begin{array}{l}\text { Proyecto ferrourbanístico en anti- } \\
\text { gua estación de ferrocarril }\end{array}$ \\
\hline $\begin{array}{l}\text { Diagnóstico desarro- } \\
\text { llo conceptual. Posa- } \\
\text { das Encarnación }{ }^{39}\end{array}$ & 2005 & $\begin{array}{l}\text { Posadas (Argen- } \\
\text { tina) y Encarna- } \\
\text { ción (Paraguay) }\end{array}$ & $\begin{array}{l}\text { Empresa Binacional Yaciretá } \\
\text { (EBY) }\end{array}$ & Convenio marco & 9 ha. & $\begin{array}{l}\text { Diagnóstico expeditivo - propuesta } \\
\text { de desarrollo conceptual }\end{array}$ \\
\hline $\begin{array}{l}\text { Convenio marco con } \\
\text { Municipalidad de } \\
\text { Morón }\end{array}$ & 2005 & $\begin{array}{l}\text { Morón Provincia } \\
\text { de Buenos Aires }\end{array}$ & Municipalidad de Morón & Convenio marco & & $\begin{array}{l}\text { Asistencia técnica en desarrollo } \\
\text { urbano en diversas áreas del } \\
\text { municipio }\end{array}$ \\
\hline $\begin{array}{l}\text { Convenio Provincia de } \\
\text { Corrientes }\end{array}$ & 2006 & Corrientes & $\begin{array}{l}\text { Gobierno de la Provincia de } \\
\text { Corrientes }\end{array}$ & Convenio marco & & $\begin{array}{l}\text { Asistencia técnica para revitali- } \\
\text { zación de Costanera de ciudad de } \\
\text { Corrientes y visión de ciudad. }\end{array}$ \\
\hline $\begin{array}{l}\text { Plan Maestro de } \\
\text { Resistencia }\end{array}$ & 2011 & $\begin{array}{l}\text { Resistencia } \\
\text { (Chaco) }\end{array}$ & $\begin{array}{l}\text { Acuerdo con Fiduciaria del } \\
\text { Norte S.A. }\end{array}$ & Acuerdo & $150 \mathrm{ha}$. & $\begin{array}{l}\text { Plan Maestro de Urbanización. } \\
\text { Addenda con el objeto de ampliar el } \\
\text { proyecto inicial }\end{array}$ \\
\hline $\begin{array}{l}\text { Convenio Coopera- } \\
\text { ción con Quilmes }\end{array}$ & 2012 & $\begin{array}{l}\text { Quilmes, Provin- } \\
\text { cia de Buenos } \\
\text { Aires }\end{array}$ & $\begin{array}{l}\text { Municipalidad } \\
\text { de Quilmes }\end{array}$ & $\begin{array}{l}\text { Convenio marco } \\
\text { de cooperación }\end{array}$ & & Desarrollar proyectos urbanísticos \\
\hline
\end{tabular}

Fuente: elaboración propia a partir de balances de la Corporación Antiguo Puerto Madero (2001 al 2012), publicaciones de la misma (Corporación Antiguo Puerto Madero, 2011) y artículos de diario nacionales y provinciales. 


\section{TABLA 3. LA EXPANSIÓN DE LA CORPORACIÓN ANTIGUO PUERTO MADERO: INICIATIVAS EN CIUDADES DE AMÉRICA LATINA.}

\begin{tabular}{|c|c|c|c|c|c|c|}
\hline Iniciativas & Año & Ubicación & Comitente/Socios & $\begin{array}{l}\text { Tipo de } \\
\text { acuerdo }\end{array}$ & Tamaก̃o & Tareas \\
\hline $\begin{array}{l}\text { Ciudad colonial de } \\
\text { Santo Domingo }\end{array}$ & 2002 & $\begin{array}{l}\text { República } \\
\text { Dominicana }\end{array}$ & $\begin{array}{l}\text { Unidad Técnica Plan } \\
\text { de Revitalización de la } \\
\text { Ciudad Colonial } \\
\text { Consorcio con: Garay } \\
\text { y Asoc. Moscato } \\
\text {-Schere }\end{array}$ & $\begin{array}{l}\text { Licitación } \\
\text { Internacional }\end{array}$ & & $\begin{array}{l}\text { Seleccionado para presentar pro- } \\
\text { puesta en licitación internacional. } \\
\text { Formulación de plan estratégico y } \\
\text { diagnóstico socioeconómico }\end{array}$ \\
\hline $\begin{array}{l}\text { Franja costera ciu- } \\
\text { dad de Asunción }\end{array}$ & 2004 & $\begin{array}{l}\text { Asunción } \\
\text { - Paraguay }\end{array}$ & $\begin{array}{l}\text { Convenio con empresa } \\
\text { "Alberto Barrail e hijos } \\
\text { S.A." }\end{array}$ & Convenio & 16 ha. & $\begin{array}{l}\text { Consultoría y promoción inmobiliaria } \\
\text { y de inversiones. desarrollo de franja } \\
\text { costera de Asunción }\end{array}$ \\
\hline $\begin{array}{l}\text { Plan maestro del } \\
\text { portal bicentenario } \\
\text { - Santiago de Chile }\end{array}$ & 2004 & $\begin{array}{l}\text { Santiago de } \\
\text { Chile-Chile }\end{array}$ & $\begin{array}{l}\text { Ministerio de Urbanis- } \\
\text { mo y Vivienda de Chile. } \\
\text { CAPMSA integra un } \\
\text { consorcio adjudicatario }\end{array}$ & $\begin{array}{l}\text { Licitación } \\
\text { nacional e } \\
\text { internacio- } \\
\text { nal. Integra } \\
\text { consorcio } \\
\text { adjudicatario }\end{array}$ & 245 ha. & $\begin{array}{l}\text { Asociado en plan maestro de } \\
\text { desarrollo (diagnóstico, estudio } \\
\text { de mercado, propuesta urbana e } \\
\text { inmobiliaria, modelo de gestión, plan } \\
\text { de marketing, factibilidad sociales y } \\
\text { económica, impacto ambiental y vial } \\
\text { e implementación) }\end{array}$ \\
\hline $\begin{array}{l}\text { Diagnóstico - pro- } \\
\text { puesta de desa- } \\
\text { rrollo conceptual } \\
\text { en Posadas y } \\
\text { Encarnación }\end{array}$ & 2005 & $\begin{array}{l}\text { Posadas } \\
\text { (Argentina)y } \\
\text { Encarnación } \\
\text { (Paraguay) }\end{array}$ & $\begin{array}{l}\text { Empresa Binacional } \\
\text { Yaciretá (EBY) }\end{array}$ & $\begin{array}{l}\text { Convenio } \\
\text { marco }\end{array}$ & 9 ha. & $\begin{array}{l}\text { Diagnóstico expeditivo - propuesta } \\
\text { de desarrollo conceptual }\end{array}$ \\
\hline La Loma & 2009 & $\begin{array}{l}\text { Barranquilla } \\
\text { Colombia }\end{array}$ & $\begin{array}{l}\text { Cámara de Comercio de } \\
\text { Barranquilla. Coordina- } \\
\text { ción Arq. Moscato }\end{array}$ & Convenio & 90 ha. & $\begin{array}{l}\text { Asistencia técnica y desarrollo } \\
\text { conceptual de alternativa de urbani- } \\
\text { zación de la isla de "La Loma" }\end{array}$ \\
\hline $\begin{array}{l}\text { Convenio marco de } \\
\text { cooperación Com- } \\
\text { panhia de Desen- } \\
\text { volvimento Urbano } \\
\text { da Região do Porto } \\
\text { do Rio de Janeiro }\end{array}$ & 2012 & $\begin{array}{l}\text { Río de Janeiro } \\
\text { - Brasil }\end{array}$ & $\begin{array}{l}\text { Companhia de Desen- } \\
\text { volvimento Urbano da } \\
\text { Região do Porto do Rio } \\
\text { de Janeiro }\end{array}$ & $\begin{array}{l}\text { Convenio } \\
\text { marco de } \\
\text { cooperación }\end{array}$ & 500 ha. & $\begin{array}{l}\text { Implementar una política activa en } \\
\text { materia de desarrollo urbano en la } \\
\text { región portuaria de Río de Janeiro }\end{array}$ \\
\hline
\end{tabular}

Fuente: elaboración propia a partir de balances de la Corporación Antiguo Puerto Madero (2001 al 2012), publicaciones de la misma (Corporación Antiguo Puerto Madero, 2011) y artículos de diarios latinoamericanos. 


\section{TABLA 4. LA EXPANSIÓN DE LA CORPORACIÓN ANTIGUO PUERTO MADERO: INICIATIVAS EN CIUDADES FUERA DE AMÉRICA LATINA.}

\begin{tabular}{|c|c|c|c|c|c|c|}
\hline Iniciativas & Año & Ubicación & Comitente / Socios & $\begin{array}{l}\text { Tipo de } \\
\text { acuerdo }\end{array}$ & Tamaño & Tareas \\
\hline Estación Norte & 2002 & Osaka-Japón & $\begin{array}{l}\text { Corporación de Desarrollo } \\
\text { Urbano - Corporación } \\
\text { pública de Construcción del } \\
\text { Ferrocarril Japonés - Fede- } \\
\text { ración Económica de Kan- } \\
\text { sai - Cámara de Comercio } \\
\text { e Industria y Gobierno de } \\
\text { Osaka. Estudio asociado: } \\
\text { Mario Roberto Álvarez }\end{array}$ & $\begin{array}{l}\text { Concurso. } \\
\text { Premio a la } \\
\text { excelencia por } \\
\text { la propuesta } \\
\text { presentada }\end{array}$ & 26 ha. & $\begin{array}{l}\text { Concurso internacio- } \\
\text { nal de ideas - remo- } \\
\text { delación de estación } \\
\text { ferroviaria de cargas }\end{array}$ \\
\hline $\begin{array}{l}\text { Waterford } \\
\text { North Quays }\end{array}$ & 2002 & Irlanda & $\begin{array}{l}\text { Oficina de Gobierno - Ofi- } \\
\text { cina de Trabajos Públicos } \\
\text { del Instituto Real de Arqui- } \\
\text { tectos de Irlanda. Estudio } \\
\text { asociado: Becker - Ferrari }\end{array}$ & Concurso & 3 ha. & $\begin{array}{l}\text { Concurso internacio- } \\
\text { nal de arquitectura } \\
\text { y diseño urbano. } \\
\text { Formular Plan Maes- } \\
\text { tro para readecua- } \\
\text { ción de Costa Norte } \\
\text { de Waterford City } \\
\text { y diseño de edificio } \\
\text { multifuncional }\end{array}$ \\
\hline $\begin{array}{l}\text { Memphis } \\
\text { Waterfront }\end{array}$ & 2002 & $\begin{array}{l}\text { Mepmphis } \\
\text { - USA }\end{array}$ & $\begin{array}{l}\text { Riverfront Development } \\
\text { Corporation de Memphis. } \\
\text { Estudio asociado: Magari- } \\
\text { ños y Rivoira }\end{array}$ & Concurso & 2,3 ha. & $\begin{array}{l}\text { Concurso interna- } \\
\text { cional de proyectos } \\
\text { para desarrollo del } \\
\text { nuevo masterplan } \\
\text { para conectar ciudad } \\
\text { y río Missisipi }\end{array}$ \\
\hline
\end{tabular}

Fuente: elaboración propia a partir de balances de la Corporación Antiguo Puerto Madero (2001 al 2012) y publicaciones de la misma (Corporación Antiguo Puerto Madero, 2011). 
Las propuestas promovidas por la CAPMSA no circulan en cualquier dirección. Desde la perspectiva aquí presentada, entender su direccionalidad supone considerar las relaciones -y contactos previos- que colaboran en tal sentido. En efecto, como fue indicado, dentro del corpus de convenios con ciudades argentinas, la ciudad de Buenos Aires es la que cuenta con una mayor presencia, sobre todo en los primeros años de expansión de la Corporación. Esto obedece a diversos motivos: la propia CAPMSA cuenta con representantes del Gobierno de la Ciudad Autónoma de Buenos Aires (GCBA), lo cual facilita los contactos a la hora de generar convenios con tal gobierno. Así, el abordaje de las trayectorias de integrantes de la CAPMSA permite señalar que hacia el año 2000, Roberto Converti, a cargo de la Corporación, venía de ocupar el cargo de Subsecretario de Planeamiento Urbano del GCBA. Dicho cruce se reitera con otros integrantes de la Corporación como Enrique Fazio, quien previamente a ocupar un lugar en la Corporación estuvo a cargo de la Secretaría de Planeamiento Urbano y Medio Ambiente del GCBA ${ }^{39}$.

Asimismo, varios de los convenios que refieren a la ciudad de Buenos Aires tienen que ver con terrenos

39 Sin embargo, en casos como el del Proyecto Retiro, estos cruces entre integrantes de la CAPMSA y el GCBA no garantizan el acuerdo entre dicho gobierno y el Gobierno Nacional a la hora de definir y materializar las propuestas promovidas por la Corporación (Cuenya, 2006). adyacentes al área de Puerto Madero (localización del Puerto de Cruceros, Isla Demarcchi, conexión Puerto Madero - La Boca). Esto no responde a una cuestión meramente de localización. Tal como lo revelan algunos informantes clave vinculados a la Corporación, en varias oportunidades la propia CAPMSA moviliza estudios y relaciones a los fines de ser convocada por el GCBA, demostrando así su interés por expandir sus límites en las zonas próximas al área inicial de influencia. A su vez, dado que una parte relevante de esas iniciativas se vinculan a áreas en las cuales tanto el Gobierno Nacional como el GCBA tienen incumbencia legal -el primero en tanto propietario de tierras y el segundo en tanto encargado de otorgar normativa a las mismas-, la Corporación se auto promueve como una herramienta útil a los fines de coordinar tal interjurisdiccionalidad, al estar integrada por representantes de ambos gobiernos ${ }^{40}$.

Por otro lado, la provincia de Mendoza cuenta con una importante presencia (Madero-Mendoza, Maipú, San Rafael, Potrerillos) en la expansión de la Corporación. La proliferación de convenios e iniciativas entre la CAPMSA y Mendoza se explica, entre otros factores, por la presencia de directivos

40 Luego, en contextos de disputas entre ambos gobiernos, la fortaleza de la CAPMSA -estar integrada por ambos gobiernos-se transforma en una limitación en tanto tierras del Gobierno Nacional en Buenos Aires serán gestionadas sin la mediación de la Corporación, para limitar el protagonismo que el gobierno de la ciudad pueda tener en tales asuntos. 
de la Corporación provenientes y con experiencia política en tal provincia, como es el caso de Alfredo Porras. Así, puede reiterarse cómo la revisión de las trayectorias de los integrantes de la Corporación que intervienen en el proceso de su expansión, permite dar cuenta de la multiplicidad de direcciones que ésta toma.

Tanto en concursos como en convenios, la Corporación actúa en conjunto con diversos estudios de arquitectura de Buenos Aires. Esto nos permite advertir la variedad de circuitos de movilidad de políticas urbanas, así como las múltiples escalas y actores que intervienen en estos procesos. Asimismo, aquella expansión permite observar diversos cruces entre la CAPMSA y ex integrantes de la misma, reafirmando la utilidad del abordaje de trayectorias en el análisis de su expansión. Tal es el caso de Alfredo Garay, quien fue Secretario de Planeamiento Urbano durante el inicio del proyecto Puerto Madero e integrante de la CAPMSA entre 1989 y 1992 y desde de 2008. Por eso, su participación en el concurso de Santo Domingo, en el año 2002, se produce mientras se encuentra fuera de la Corporación. En tanto, cabe recordar los lazos existentes con el arquitecto Moscato, quien acompaña la gestión de Garay en los años iniciales del proyecto Puerto Madero como consejero de planificación de la Municipalidad de la ciudad de Buenos Aires, y será parte de iniciativas como el concurso de Santo Domingo y 'La Loma' en Barranquilla, a partir del año 2009.

Aun así, estas relaciones no agotan la multiplicidad y variedad de escalas y circuitos de movilidad de políticas y modelos urbanos asociados a la Corporación y a la experiencia de Puerto Madero. Tal como lo indican Cochrane y Ward ${ }^{41}$, pensar en términos de la movilidad de las políticas requiere entender la multiplicidad y el entrecruzamiento de los espacios de su elaboración. En efecto, como ramificación o derivación de estos procesos aunque por fuera de la Corporación, y como entrecruzamiento entre ámbitos públicos y privados cabe señalar el rol que asumen otros ex integrantes de la misma. Así, puede mencionarse el caso del aludido Roberto Converti, quien tras abandonar la presidencia de la CAPMSA monta en 2002 una oficina de consultoría urbana (OFICINA URBANA). Desde allí se concentra en la temática de asesorías y consultorías en remodelación de áreas portuarias o bordes de ríos en distintas ciudades (como Rosario, Santa Fe, Neuquén ${ }^{42}$, Salta y Villa del Chocón en Argentina), retomando el know how desplegado

41 Cochrane y Ward, 2012.

42 Participando en la creación de nuevas corporaciones de desarrollo urbano como la Corporación para el Desarrollo Integral de Neuquén Sociedad del Estado (CORDINEU-SE) y dirigiendo el plan maestro para el desarrollo del 'Paseo de la Costa'. 
en la Corporación y aprovechando ciertas relaciones allí tejidas. Tales relaciones le permitirán ser parte de redes de ciudades portuarias. Es decir, espacios donde se yuxtapone la promoción de determinadas políticas urbanas y el desarrollo de conocimientos sobre tales asuntos a partir, por ejemplo, de congresos y de la elaboración de textos y manuales sobre ciudades portuarias. Esos vínculos previos también facilitarán el acceso a un mercado internacional de consultoría. Así, Converti accederá a cargos como el de consultor externo de organismos internacionales como URB-AL. Una dinámica relativamente análoga puede indicarse a propósito de Guillermo Suaya, otro ex integrante de la CAPMSA que pocos años después de su paso por la misma integra el consorcio de profesionales de Ecuador, Perú, Inglaterra y Argentina que lleva adelante la renovación de la costanera de Guayaquil "Malecón 2000"43. De esta forma, tales relaciones previas y el know-how específico adquirido durante el pasaje por la Corporación serán también reutilizados y capitalizados en trayectorias laborales personales.

Cambiando el prisma de análisis cabe indicar, a lo largo del tiempo, una serie de modificaciones respecto al rol que asume, o busca asumir, la Corporación y a las utilidades económicas esperadas en las diferentes iniciativas. Este proceso, da cuenta

43 Santos, 2000. de los aprendizajes que la CAPMSA adquiere y transmite en su experiencia de expansión y movilidad de políticas y modelos urbanos. Es decir, los procesos de aprendizaje también son multidireccionales. Por un lado, diversas ciudades entran en contacto con la CAPMSA y aprenden de Puerto Madero -buscando legitimar en contextos locales sus propuestas, movilizando la referencia a casos 'exitosos'-. Como contrapartida, la Corporación aprende de su propia experiencia de expansión. Así, por ejemplo, en el convenio Mendoza-Madero las utilidades se producirían una vez terminado el proyecto de remodelación de la antigua estación de ferrocarril. Sin embargo, el mismo no se materializó, tanto por resistencias de actores locales $^{44}$ como por disputas intra-estatales entre la Corporación y el Organismo Nacional de Bienes del Estado (ONABE), propietario de los terrenos ${ }^{45}$. Así, la CAPMSA no obtiene ganancias económicas sino pérdidas, al asumir el pago correspondiente al proyecto elaborado por el estudio de arquitectura 'Mario Roberto Álvarez'. Por el contrario, en convenios posteriores, las utilidades de la Corporación se estipulan en distintas etapas: antes y después del desarrollo del proyecto. De esta manera puede señalarse un proceso de aprendizaje de la Corporación con experiencias de convenios que no se materializan y a partir de los cuales la CAPMSA ya no buscará proponerse única ni centralmente como constructora

44 Salomone y Marsonet, 2011.

45 Corporación Antiguo Puerto Madero, 2009. 
o desarrolladora de proyectos sino también asumiendo un rol de consultora, entendiendo que las dinámicas posteriores de los convenios escapan a su control.

Sin embargo, como ya se indicó, más allá de los -escasos- ingresos obtenidos, la posibilidad de expandirse por parte de la Corporación garantiza su continuidad institucional una vez que ésta concluya la urbanización de Puerto Madero, algo que no parece lejano en el tiempo dado su alto grado de avance. A su vez, permite que actores asociados a la misma se integren a diversos circuitos de circulación de modelos urbanos, sea como socios directos de la propia Corporación o como ex integrantes de la misma que aprovechan las relaciones y el saber hacer allí adquirido y se insertan en un mercado trasnacional de consultorías privadas. Asimismo, junto al rédito económico de los convenios y la prolongación de sus funciones, la CAPMSA busca consolidar su imagen en el exterior "como un exitoso desarrollador urbano en gran escala"46, algo que, se espera, fortalezca al mismo tiempo la posibilidad de obtener nuevos encargos y mayor legitimidad dentro del país.

46 Corporación Antiguo Puerto Madero, 2009, p. 7

\section{Más allá de los convenios: la multiplicidad de circuitos de movilidad}

Diversos actores buscan en un contexto internacional políticas urbanas que puedan serles de ayuda en términos tanto prácticos como políticos. Asimismo, las políticas 'importadas' desde otros lugares arriban a los contextos locales con un plus de prestigio ${ }^{47}$. Así, cambiando el prisma de observación y parafraseando lo que González ${ }^{48}$ expresarespecto a ciudades como Barcelona y Bilbao, cabe señalar que si los expertos o políticos de las ciudades mencionadas en las tablas 1, 2 y 3 establecen convenios con la CAPMSA o visitan Puerto Madero a los fines de aprender de su experiencia $-y$ legitimar diversos proyectos urbanos en contextos locales apelando al supuesto éxito de la experiencia de referencia-, esto ocurre porque previamente han oído o leído respecto de la misma a través de la prensa, literatura especializada, documentos de políticas, redes de ciudades, exhibiciones o muestras donde se publicita y relata la operación. Es decir que la expansión, la circulación y la movilidad de la experiencia de Puerto Madero exceden tanto al aludido corpus de convenios como a los circuitos a través de los cuales éstos se despliegan.

47 Temenos y Mc Cann, 2012.

48 González, 2011. 
El proyecto Puerto Madero así como la CAPMSA han recibido múltiples premios y reconocimientos internacionales y han sido exhibidos en diversas muestras y presentados en distintos encuentros, a partir de instituciones vinculadas al universo de la arquitectura, el urbanismo y la gestión urbana del sector inmobiliario. A su vez, la CAPMSA integró diferentes redes (como la Asociación Internacional de Villas y Puertos), se ha promovido a sí misma a partir de publicaciones (de la propia CAPMSA como de integrantes y ex integrantes de la misma) y del sitio web propio -en varios idiomas- (www. puertomadero.com), participó de los mencionados concursos internacionales, comenzó a ser estudiada como ejemplo de best practice en múltiples programas universitarios de urbanismo y planificación urbana y fue y es objeto de tesis e investigaciones tanto críticas como apologéticas.

Investigadoras como Sánchez y Moura ${ }^{49}$ sostienen que los documentos oficiales de agencias multilaterales de desarrollo juegan un rol fundamental a la hora de codificar experiencias como best practices y favorecer su internacionalización y reproducción

49 Sánchez y Moura, 2005. en tanto 'modelo'. Cabe destacar que el proyecto de Puerto Madero fue incluido como ejemplo en múltiples libros y manuales, entre los que se destaca aquel publicado por Borja y Castells ${ }^{50}$, producido en el marco de la Conferencia de Naciones Unidas sobre Asentamientos Humanos (Habitat II, realizada en Estambul en 1996) y traducido a múltiples idiomas. Retomando los entrecruzamientos y la multidireccionalidad de los circuitos de movilidad de las políticas y modelos urbanos y la utilidad del abordaje de trayectorias, cabe agregar que el propio Jordi Borja fue uno de los expertos que en 1990 intervino en una propuesta catalana para Puerto Madero: el Plan Estratégico Antiguo Madero $^{51 y 52}$. Por aquellos años, Borja estaba a cargo de Tecnologías Urbanas de Barcelona Sociedad Anónima (TUBSA), una empresa público-privada que buscaba exportar tecnología urbana catalana. Tanto con TUBSA como en la consultora privada que inaugura en 1995 (URBANTC - Jordi Borja, Urban Technology Consulting), Borja recorrerá América Latina asesorando y produciendo planes estratégicos en múltiples ciudades ${ }^{53}$. Así, Borja reaparece

\footnotetext{
50 Borja y Castells, 1997.

51 Borja, 1990; Consultores Europeos Asociados, 1990.

52 Presentada en 1990, la propuesta catalana para Puerto Madero fue rápidamente dejada de lado dada la resistencia de diversos actores locales. Un abordaje de tal asunto puede encontrarse en Gorelik, 2007.

53 Si bien surgidos en el marco de contextos políticos previos, Borja mantenía relaciones académicas y políticas con expertos argentinos -y latinoamericanos - desde la década del setenta (Jajamovich, 2012).
} 
en esta historia de la circulación y movilidad de la operación Puerto Madero ocupando lugares diversos en distintos contextos. Se reitera entonces aquella lógica social que indica que cada vez que se crean grupos y se mantienen lazos de solidaridad, los objetivos pueden cambiar, pero su funcionamiento y su capacidad organizativa permanecen ${ }^{54}$.

\section{Conclusiones}

Hemos descrito el modo en que desde el año 1999 en adelante, la CAPMSA se ha expandido de diversas formas a lo largo de múltiples ciudades de la región. En ese marco, se ha dialogado con un creciente campo de estudio centrado en los viajes, la transferencia y el flujo de los modelos urbanos, políticas urbanas, ideas y técnicas, así como con algunos abordajes respecto a los grandes proyectos urbanos en América Latina.

En relación al primer eje, el abordaje de la circulación de la Corporación permite ampliar el estudio de la movilidad de las políticas y modelos urbanos más allá de su circulación entre ciudades del norte y entre ciudades del norte y del sur, incrementando un conjunto de investigaciones que rastrean tal circulación entre ciudades de la región, es decir, en circuitos Sur - Sur. Paralelamente a la expansión de la CAPMSA y los convenios que ésta produce con distintos comitentes, integrantes y ex integrantes de la misma hacen uso de relaciones producidas en su interior así como del know how allí desplegado. De ese modo se ramifican y yuxtaponen los circuitos de circulación de políticas y modelos urbanos tornándolos multidireccionales y dificultando una rígida delimitación. Así, cabe reiterar el aporte que supone el abordaje de las trayectorias de integrantes de la CAPMSA a la hora de entender tanto la circulación de la Corporación como sus derivaciones.

Tal como fue señalado, las políticas urbanas no circulan en cualquier dirección. Su circulación supone el trabajo de actores interesados en movilizar esos procesos. Entre los sentidos que se han indicado a propósito de la circulación de la Corporación y la proliferación de convenios, se encuentra el rédito económico que la CAPMSA espera obtener. Sin embargo, no parecen haber rendido los frutos esperados en tanto gran parte de los convenios aún no se materializaron y no derivaron en ingresos a la Corporación. Más allá de esto, cabe reiterar que tal expansión le ha permitido a la Corporación su continuidad institucional una vez que se 'libera' de la función exclusiva de urbanizar las 170 hectáreas de Puerto Madero. Esto le habilita un 'futuro' que no se encuentra atado al devenir de tal área, cuyo final no parece ser tan lejano dada la escasez de emprendimientos que quedan por realizar en su

Donatello, 2003. 
perímetro. La expansión de la Corporación también busca consolidar su imagen en el exterior ${ }^{55}$, algo que, se espera, fortalezca tanto la posibilidad de obtener nuevos encargos como su inserción en el contexto institucional argentino.

A su vez, en un cruce que da cuenta de la permeabilidad entre lógicas públicas y privadas, se puede añadir que la actividad de la Corporación por fuera del perímetro de Puerto Madero también facilita la obtención de ganancias materiales y simbólicas para una serie de actores que se asocian a tales procesos, como esos estudios de arquitectura y de urbanismo, consultores privados, etc. En ese sentido, el abordaje de las trayectorias de integrantes, ex integrantes y socios de la CAPMSA en los años analizados ha permitido dar cuenta de la multiplicidad y yuxtaposición de circuitos de movilidad de las políticas urbanas. Asimismo, ha permitido establecer conexiones concretas entre Puerto Madero y la renovación de la costanera de Guayaquil y ha posibilitado dar cuenta de la multiplicidad de cruces que la CAPMSA produce tanto con oficinas privadas (como la dirigida por Converti que derivará en iniciativas en otras ciudades), como con consultores y empresas extranjeras de consultorías (como es el caso de Jordi Borja, TUBSA y URBANTC - Jordi Borja Urban Technology Consulting, que reaparece en distintos momentos y de distintas maneras en la vida institucional de la Corporación).

En segundo lugar, la perspectiva presentada buscó dialogar y ampliar el conjunto de procesos y actores iluminados por diversas investigaciones existentes respecto a los grandes proyectos urbanos en América Latina. Entre otros aspectos, esto fue posible a partir de diversos desplazamientos. En primer lugar, se postula que el abordaje de políticas urbanas puede complejizarse analizando no sólo sus efectos -urbanos, sociales, económicos, políticos, inmobiliarios, etc.-, sino también a partir del análisis de cómo se conforman, quiénes intervienen en tal conformación, etc. Así, se ha presentado un enfoque que busca contribuir a dar cuenta de la multiplicación de intervenciones urbanas similares en diversas ciudades pero ampliando las escalas de análisis más allá de los estudios de casos nacionales y/o locales. En ese sentido, el abordaje de la circulación internacional de políticas urbanas y de expertos ha permitido iluminar algunos de los actores concretos que intervienen en tales procesos y señalar que lejos de ser el mero producto del accionar del capital global en las ciudades, la multiplicación de grandes proyectos urbanos puede abordarse considerando otros actores y/o procesos.

Abordar la circulación de la corporación desde 1999 hasta nuestros días, ha posibilitado conocer 
distintos aprendizajes. Por un lado, el de diversas ciudades que aprenden de Puerto Madero buscando legitimar proyectos urbanos locales invocando el 'éxito' y el respaldo de una 'best practice', aunque, como ocurrió en diversos casos y por variados motivos, la apelación a tal experiencia no garantice la materialización de los proyectos promovidos. Por otro lado, el de la propia CAPMSA aprendiendo de su misma experiencia de expansión. En este último sentido, se ha apuntado cómo la CAPMSA reformula algunas características de los convenios en los que va participando. También el caso donde las utilidades dejan de contemplarse únicamente en relación a la finalización de los proyectos y se incorporan en distintas etapas de los mismos, previendo la eventualidad de la no materialización de las propuestas. Además, se han señalado algunas variaciones en la participación de la Corporación en los convenios, asumiendo en reiteradas ocasiones el rol de consultor en tanto la propia experiencia demuestra la dificultad de asumir el papel de desarrollador y de controlador de las dinámicas locales que los convenios despliegan.

Las narrativas hegemónicas respecto a los grandes proyectos urbanos dejan de lado aspectos que oscurecen los relatos exitosos. Más allá de las variaciones observables en las ecuaciones de participación entre actores públicos y privados, los grandes proyectos urbanos, como el de Puerto Madero, modifican la estructura de la centralidad de las ciudades. Así, cambian la rentabilidad de los usos del suelo, modifican los mecanismos de gestión pública y alteran en términos funcionales y físico espaciales áreas centrales estratégicas. Entre otras, pueden recuperarse tres críticas que recibiera la operación Puerto Madero: tierras y edificios públicos, pertenecientes a la ciudadanía, fueron vendidas a un reducido grupo de promotores, inversores y usuarios de altos ingresos; las plusvalías captadas por el sector público fueron aplicadas al mismo sitio, beneficiando al aludido reducido grupo y reforzando la diferenciación respecto a otros barrios de la ciudad; la propia CAPMSA fue cuestionada por no rendir cuentas de su accionar frente a la ciudadanía ${ }^{56}$. Se trata de críticas que no están en los relatos de los 'importadores' y 'exportadores de estas experiencias. Sin embargo, tales críticas sí son retomadas por diversos movimientos sociales y sectores profesionales y políticos que en distintas ciudades se oponen o discuten proyectos inspirados en iniciativas como Puerto Madero. Por último, cabe señalar que la circulación de políticas urbanas no es un proceso que se reduzca meramente a la circulación de iniciativas urbanas de orientación neoliberal. En efecto, la perspectiva aquí presentada puede ser retomada para entender tanto la circulación de políticas que promuevan lógicas de intervención alternativas como para abordar la circulación de perspectivas críticas y resistencias a las políticas neoliberales.

56 Cuenya, 2012, p. 55. 


\section{Bibliografía}

ARANTES, Otilia; VAINER, Carlos y MARICATO, Ermínia. A cidade do pensamento único. Desmachando consensos. Petrópolis, Brasil, Vozes. 2000. 192 p. ISBN 85-326-2384-0.

BAIOCCHI, Gianpaolo y GANUZA, Ernesto. Participatory budgeting as If emancipation mattered. Politics E Society. 42(1): 29-50, 2014. ISSN 00323292. DOI 10.1177/0032329213512978.

BORJA, Jordi. Presentación. En: CONSULTORES Europeos Asociados. Plan estratégico antiguo Puerto Madero. Buenos Aires, Argentina, [s.n.]. 1990. p. 9-14.

BORJA, Jordi y CASTELLS, Manuel. Local y global: la gestión de las ciudades en la era de la información. Madrid, España, Taurus. 1997. 418 p. ISBN 84-306-0269-0.

BRITES, Walter. La mega-hidroeléctrica Yacyretá en el vórtice de las reconfiguraciones urbanas. El caso de las ciudades de Posadas, Argentina, y Encarnación, Paraguay. URBS. Revista de Estudios Urbanos y Ciencias Sociales. 4(2): 91-107, julio 2014. ISSN 2014-2714.

CICCOLELLA, Pablo. Globalización y dualización en la región metropolitana de Buenos Aires. Grandes inversiones y reestructuración socioterritorial en los años noventa. EURE. 25(76): 5-27, diciembre 1999. ISSN 0250-7161. DOI 10.4067/ S0250-71611999007600001.
COCHRANE, Allan y WARD, Kevin. Researching the geographies of policy mobility: confronting the methodological challenges. Environment and Planning A. 44(1): 5-12, enero 2012. ISSN 0308-518X. DOI 10.1068/a44176.

CONSULTORES Europeos Asociados. Plan estratégico antiguo Puerto Madero. Buenos Aires, Argentina, [s.n.]. 1990. $3 \mathrm{v}$.

CORPORACIÓN Antiguo Puerto Madero S.A. Puerto Madero. 1989-2011. [En línea]. Puerto Madero. Octubre, 2011. [Fecha de consulta: 14 abril 2014]. Disponible en: http://www.puertomadero.com/ bitacora/Default.html.

--- Balances. [En línea]. Puerto Madero. 2001, 2002, 2003, 2004, 2005, 2006, 2007, 2008, 2009, 2010, 2011 y 2012. Disponible en: http://www.puertomadero.com/balances_esp.php.

--- 1989-1999, un modelo de gestión urbana. Buenos Aires, Argentina, Larivière. 1999. 125 p. ISBN 987-9395-03-4.

CUENYA, Beatriz. Grandes proyectos urbanos, cambios en la centralidad urbana y conflictos de intereses. Notas sobre la experiencia argentina. En: CUENYA, Beatriz, comp.; NOVAIS, Pedro, comp. y VAINER, Carlos, comp. Grandes proyectos urbanos. Miradas críticas sobre la experiencia argentina y brasilera. Buenos Aires, Argentina, Café de las Ciudades. 2012. p. 27-66. ISBN 978-987-25706-3-7.

--- Large urban projects and social actors, forces supporting and opposing the production process of 
the Retiro project 1991-2001. Delft, Delft University of Technology. 2006. Tesis doctoral. 235 p.

CUENYA, Beatriz y CORRAL, Manuela. Empresarialismo, economía del suelo y grandes proyectos urbanos: el modelo de Puerto Madero en Buenos Aires. EURE. 37(111): 25-45, mayo 2011. ISSN 02507161. DOI 10.4067/S0250-71612011000200002.

DONATELLO, Luis. Religión y política: las redes sociales del catolicismo post-conciliar y los Montoneros, 1966-1973. Estudios Sociales. 13(24): 89-112, enero 2003. ISSN 0327-4934.

ETULAIN, Juan Carlos. ¿Gestión promocional o privatización de la gestión urbanística? Proyecto urbano Puerto Madero, Buenos Aires, Argentina. Bitácora Urbano Territorial. 1(12): 171-184, enero 2008. ISSN 0124-7913.

GONZÁLEZ, Sara. Bilbao and Barcelona 'in motion'. How urban regeneration 'models' travel and mutate in the global flows of policy tourism. Urban Studies. 48(7): 1397-1418, mayo 2011. ISSN 00420980. DOI 10.1177/0042098010374510.

GARAY, Alfredo. On the administration of urban projects: the lessons of Puerto Madero. En: LIERNUR, Jorge, ed. Puerto Madero waterfront. Munich, Alemania, Prestel. 2007. p. 74-83. ISBN 978-3-7913-3517-9.

GORELIK, Adrián. The Puerto Madero competition and urban ideas in Buenos Aires in the 1980s. En: LIERNUR, Jorge, ed. Puerto Madero waterfront.
Múnich, Alemania, Prestel. 2007. p. 62-73. ISBN 978-3-7913-3517-9.

HARRIS, Andrew y MOORE, Susan. Planning histories and practices of circulating knowledge. International Journal of Urban and Regional Research. 37(5): 1499-1505, septiembre 2013. ISSN 03091317. DOI 10.1111/1468-2427.12043.

HEALEY, Patsy. Circuits of knowledge and techniques: the transnational flow of planning ideas and practices. International Journal of Urban and Regional Research. 37(5): 1510-1526, septiembre 2013. ISSN 0309-1317. DOI: 10.1111/1468-2427.12044.

HEALEY, Patsy y UPTON, Robert. Crossing borders. International exchange and planning practices. Londres, Inglaterra, Routledge. 2010. 368 p. ISBN 0-415-55864-8.

JACOBS, Jane y LEES, Loretta. Defensible space on the move: revisiting the urban geography of Alice Coleman. International Journal of Urban and Regional Research. 37(5): 1559-1583, septiembre 2013. ISSN 0309-1317. DOI 10.1111/1468-2427.12047.

JAJAMOVICH, Guillermo. Miradas sobre intercambios internacionales y circulación internacional de ideas urbanas. Andamios, revista de investigación social. 10(22): 91-112, 2013. ISSN 1870-0063.

--- Revisitando Puerto Madero. CIUDADES. 24(95): 2-9, 2012. ISSN 0187-8611.

McCANN, Eugene. Urban policy mobilities and global circuits of knowledge: Toward a research agenda. Annals of the Association of American Geographers. 
101(1): 107-130, 2011. ISSN 0004-5608. DOI 10.1080/00045608.2010.520219.

McCANN, Eugene y WARD, Kevin. Urban assemblages: territories, relations, practices and power. En: McCANN, Eugene y WARD, Kevin. Mobile urbanism. Cities and policy making in the global age. Minneapolis, Estados Unidos, Minesota University Press. 2011. p. xiii-xxxv. ISBN 978-0-8166-5628-8.

McFARLANE, Collin. Learning the city: knowledge and translocal assemblage. Oxford, Inglaterra, WileyBlackwell. 2011. 214 p. ISBN 9781444343434.

MENAZZI, María Luján. Ciudad en dictadura. Procesos urbanos en la ciudad de Buenos Aires durante la última dictadura militar (1976-1983). [En línea]. Scripta Nova. Revista Electrónica de Geografía y Ciencias Sociales. 17(429), febrero, 2013. ISSN: 1138-9788. [Fecha de consulta: 19 abril 2014]. Disponible en: http://www.ub.edu/geocrit/sn/sn429.htm.

MIGNAQUI, Ileana y ELGUEZABAL, Liliana. Reforma del Estado, políticas urbanas y práctica urbanística. Las intervenciones urbanas recientes en Capital Federal: entre la ciudad global y la ciudad excluyente. En: HERZER, Hilda. Postales urbanas del final del milenio. Una construcción de muchos. Buenos Aires, Argentina, Oficina de Publicaciones del CBC. 1997. p. 219-240. ISBN 950-29-0428-1.

NASR, Joe y VOLAIT, Mercedes. Urbanism, imported or exported? Native aspirations and foreign plans.

ARTíCULO: Puerto Madero 'en movimiento': movilidad de políticas y modelos urbanos en América Latina (1999-2012)/Guillermo Paz Jajamovich
Sussex, Inglaterra, Willey Academy. 2003. 354 p. ISBN 0470851600.

NOVAIS, Pedro. Uma estratégia chamada Planejamento estratégico'. Deslocamentos espaciais e a atribuição de sentidos na teoría do planejamento urbano. Río de Janeiro, Brasil, 7 Letras. 2010. 220 p. ISBN 978-85-7577-665-0.

NOVICK, Alicia. La ciudad, el urbanismo y los intercambios internacionales. Notas para una discusión. Revista iberoamericana de urbanismo. 1(1): 4-13, enero 2009. ISSN 2013-6242.

PECK, Jamie y THEODORE, Nick. Mobilizing policy: models, methods, and mutations. Geoforum. 41(1): 169-174, enero 2010. ISSN 0016-7185.

PíREZ, Pedro. Actores sociales y gestión de la ciudad. Ciudades. 7(28): 8-14, octubre 1995. ISSN 0187-8611.

PODER Ejecutivo Nacional. Decreto NAC No 1279 / 1989. Convenio de constitución y estatuto social Corporación Antiguo Puerto Madero S.A. [En línea]. Ciudad y Derechos. Noviembre 1989. [Fecha de consulta: 15 abril 2010]. Disponible en: http://www.ciudadyderechos.org.ar/sistemas_l. php?id=40\&id2=135\&id $3=120$.

ROBIN, Silvia y VELUT, Sébastien. Entre Barcelone et Porto Alegre: la gestion municipale à Montevideo et Rosario. Géocarrefour. 80(3): 207-214, julio 2005. ISSN 1960-601X. DOI 10.4000/ geocarrefour.1191.

ROBINSON, Jennifer. The spaces of circulating knowledge. En: McCANN, Eugene y WARD, 
Kevin. Mobile urbanism: city policymaking in the global age. Minneapolis, Estados Unidos, Minesota University Press. 2011. p. 15-40. ISBN 978-0-8166-5628-8.

SALOMONE, Mariano y MARSONET, Pedro. Territorio urbano: espacio para la vida vs. espacio para el capital. La disputa por los terrenos del ferrocarril en Mendoza. Herramienta. 15(48): 153-175, octubre 2011. ISSN 1852-4710.

SÁNCHEZ, Fernanda. A reinvenção das cidades para um mercado mundial. 2 a ed. Chapecó, Brasil, Argos. 2010. 555 p. ISBN 978-85-7897-022-2.

SÁNCHEZ, Fernanda y MOURA, Rosa. Ciudadesmodelo: estrategias convergentes para su difusión internacional. Eure. 31(93): 21-34, agosto 2005. ISSN 0250-7161. DOI 10.4067/ S0250-71612005009300002.

SANTOS, Tali. ¿Quiénes dirigen la construcción del Malecón 2000? [En línea]. El Universo. 5 noviembre 2000 [Fecha de consulta: 12 octubre 2013]. Disponible en: http://www.douglasdreher.com/ publicaciones/online/ellosloestanhaciendo/ellosloestanhaciendo.htm.

SASSEN, Saskia. La ciudad global. New York, Londres, Tokio. Buenos Aires, Eudeba. 1999, 458 p. ISBN 950-23-0976-6.

TEMENOS, Cristina y McCANN, Eugene. The local politics of policy mobility: learning, persuasion, and the production of a municipal sustainability fix. Environment and Planning A. 44(6): 1389-1406, junio 2012. ISSN 0308-518X. DOI 10.1068/a44314. 